\title{
Introduction à de nouvelles recherches sur le sacrifice chez les Dogon
}

Introduction to new research on sacrifice among the Dogon

\section{Germaine Dieterlen}

\section{(2) OpenEdition}

\section{Journals}

Édition électronique

URL : http://journals.openedition.org/span/281

DOI : $10.4000 /$ span.281

ISSN : 2268-1558

Éditeur

École pratique des hautes études. Sciences humaines

\section{Édition imprimée}

Date de publication : 1 septembre 1976

Pagination : 43-50

ISSN : 0294-7080

\section{Référence électronique}

Germaine Dieterlen, «Introduction à de nouvelles recherches sur le sacrifice chez les Dogon »,

Systèmes de pensée en Afrique noire [En ligne], 2 | 1976, mis en ligne le 04 juin 2013, consulté le 20 avril 2019. URL : http://journals.openedition.org/span/281 ; DOI : 10.4000/span.281 


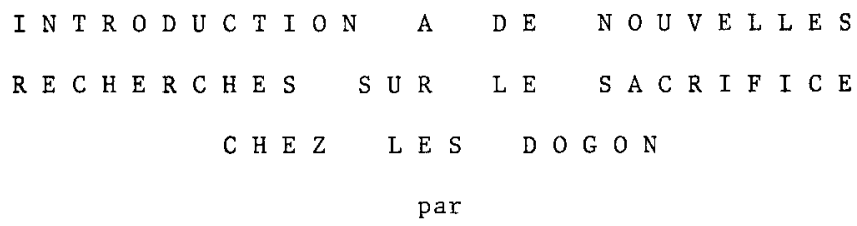

Germaine Dieterlen

Le court, mais combien pertinent, article de Marcel Griaule que nous reproduisons dans ce Cahier marque un tournant dans les recherches sur les diverses modalités du sacrifice en décrivant les mécanismes du sacrifice sanglant chez les Dogon tel qu'il est compris par les usagers. I1 relate un sacrifice précis, celui qui est offert sur les autels que 1'on peut qualifier "d'entretien de soi", car ils sont consacrés aux principes spirituels et à la force vitale d'un individu dêterminê.

L'enquête avait étê réalisêe à 1'occasion d'un événement fortuit, à propos d'un sacrifice effectué par le père d'un de nos informateurs au bénéfice de ce dernier, peu avant son mariage. L'intéressé ne procêdait pas à l'immolation, mais devait y assister pour consommer une partie du foie de la victime; s'il ne le faisait pas, le circuit lancé par $1^{\prime}$ opération resterait fermé sur lui-même ; il n'y aurait pas de dégagement de force vitale (nyoma) et l'intéressé n'en retirerait aucun profit. Il s'agissait donc ici d'un sacrifice individuel, mais les enquêtes ont confirmé que le schème observé était celui de toute opération du même genre, quels qu'en soient le ou les bénéficiaires.

Depuis la parution de cet article, un certain nombre d'informations ont été recueillies sur le terrain, non seulement par des interrogations relatives au sujet, mais aussi à l'occasion d'observations de faits rituels ou d'enquêtes menées sur d'autres aspects de la vie sociale. Nous nous proposons d'évoquer ici les étapes et les développements de ces recherches. 


\section{Germaine Dieterlen}

Tout d'abord, et. environ à la même êpoque, les enquêtes menées sur le culte des ancêtres nous avaient permis de réaliser une première analyse de la notion de personne et de ses composantes chez les Dogon. Un chapitre de notre ouvrage Les Ames des Dogon décrit notamment les autels individuels dont nous venons de parler, les caractéristiques des libations et des victimes qui leur sont offertes et le symbolisme de l'usage de ces matériaux par rapport au corps humain (1). Dans le même temps, les informations sur les devises ou "titres d'honneur" des Dogon, publiées par Solange de Ganay (2), révélaient les effets précis de leur criée sur la force vitale des individus ou des collectivités auxquels elles s'adressent.

Or notre attention, à cette époque, avait surtout été attirée par les caractéristiques et le rôle de la force vitale dont on avait dégagé, en partie, la composition, la localisation, etc... au niveau de la personne et à laquelle Marcel Griaule avait consacré un important chapitre dans son ouvrage sur les masques dogon (3). Envisagée comme une "force circulant avec le sang", elle semblait jouir d'une certaine indêpendance. Les informateurs, en effet, avaient insisté sur les effets de la libération du nycoma au moment de la mort et, bien entendu, de son agressivité en cas de mort provoquée, à la guerre, à 1 a chasse etc... Cependant, il était déjà apparu que, 1ibéré par le sacrifice, mais dirigé par la prière qui accompagnait l'immolation, le nyama d'une victime devenait l'agent d'une opération de transfert et non d'une action agressive dont il fallait se protéger.

Plus tard - également à la suite d'un événement inattendu - un certain nombre de conceptions relatives à la notion d'impuretë furent dégagées par rapport aux composantes de la personne. Les informations venaient à 1 'encontre de 1 'acception généralement admise concernant

(1) G. Dieterlen, Les Ames des Dogon, Travaux et Mémoires de l'Institut d'Ethnologie, Paris, 1941, t. XL, 268 p., 15 pl. Chapitre II (La personnalité), p. 73-91.

(2) Solange de Ganay, Les Devises des Dogon, Travaux et Mémoires de 1 'Institut d'Ethnologie, Paris, 1941, t. XLI. Chapitre VI (Remarques sur les devises et leurs effets), p. 147-176.

(3) Marcel Griaule, Masques Dogon, Travaux et Mêmoires de I'Institut d'Ethnologie, Paris, 1938, t. XXXIII, passim; et appendice : notion du nyoma p. 826-836. 
Nouvelles recherches sur le sacrifice chez les Dogon

l'impureté : celle-ci étant conçue comme un miasme ou une souillure venant, de 1 'extérieur, s'introduire dans 1 'individu, l'aute1, le lieu-dit, etc... et les perturber. Pour les Dogon, il s'agit, tout au contraire, d'une fuite de la force vitale - le mot n'est pas trop fort (4). Toute rupture d'interdit provoque cette fuite qui rend le coupable impur, c'est-à-dire un "manque" qui entraîne la désagrégation de sa personne et nuit gravement à sa stabilité. L'impureté, pum, a pour contraire omo, "vivant" : est pur ce qui est vivant. Toute impureté due à la fuite du nyama provoque un "vide" et, parallèlement, une dégradation des forces vives, de la santé, de l'êquilibre, de 1 'activité, etc... Dans certains cas graves, si une purification n'intervient pas à temps ou qu'elle n'est pas acceptée par la personnalitê lésée à cause de la rupture d'interdit (ancêtres mythiques ou familiaux généralement), celle-ci peut conduire le coupable à la mort.

on comprend mieux dès lors 1 'une des fonctions du sacrifice: le courant établi par 1 'écoulement du sang d'une victime et le dégagement de nyoma qui l'accompagne peut pallier le "manque" dont souffre l'intéressé et 1'aider à combler les pertes de substance qu'il a subies.

Une nouvelle orientation se fit jour lorsque les Dogon décidèrent d'aborder, dans leurs réponses à nos questions, l'interprétation des faits dans la perspective de l'initiation et des enseignements progressifs qu'elle implique - enseignements conçus comme autant de "paroles" de plus en plus explicites. Les premières informations recueillies par Marcel Griaule, consignées dans Dieu d'eau, soulignent qu'il convient d'envisager les modalités et les effets du sacrifice en regard de la cosmologie de cette population d'une part, dans leurs relations étroites avec les caractéristiques de la "parole", du Verbe tel qu'il est conçu par les Dogon, d'autre part. Toute opération sacrificielle met certes en branle les forces vitales des agents qui sont en cause (sacrifiant, victime, puissance surnaturelle, etc...), mais cette action est conçue fondamentalement comme une réorganisation de 1 'univers devant instaurer 1 'ère d'une "nouvelle parole" (5).

(4) Germaine Dieterlen, "Mécanisme de 1'impureté chez les Dogon", Journal de la Société des Africanistes, t. XVII, 1947, p. 81-90.

(5) Marcel Griaule, Dieu d'eau. Entretiens avec Ogotemmêli. Paris, Editions du Chêne, 1948, p. 69-76. 
Dans cet ouvrage, des développements sont consacrés au sacrifice du Nommo, "mâttre" de la parole, à sa résurrection, aux buts et effets de ces opérations, puis, parallèlement, au sacrifice de l'ancêtre Lébé, particulièrement associé aux travaux agricoles devenus indispensables pour permettre le développement de la vie des hommes sur la Terre (6). Sur un autre plan, les Dogon apportaient également des informations sur les effets de diffusion et sur les vertus du sacrifice sanglant en général. Commè pour celui du Nommo qui fut réalisê au bénéfice de toute l'humanité existante et à venir, toute opération sacrificielle poursuit ses effets, bien au-delà du moment où elle est réalisée, sur les membres de la communauté à laquelle appartient le sacrifiant : 1a "parole" qu'elle met en branle agit durablement pour le bien de tous ceux qu'elle touche (7).

Les enquêtes poursuivies dans cette nouvelle perspective permirent de mettre à jour 1 'ensemble des composantes de la personne en révélant tout d'abord le rôle vital du contenu claviculaire. Tout individu porte dans ses clavicules les symboles des huit graines de céréales réalisêes par Dieu, qui sont aussi ceux de ses nourritures de base. E1les rappellent dans le corps de 1 'homme 1 'une des étapes fondamentales de la genèse : la création de la matière vivante. Toute impureté fait fuir les graines de l'intéressé en même temps que sa force vitale (8).

D'autre part furent dëgagés le nombre exact, le symbolisme et les fonctions des principes spirituels que nous avons, pour plus de commodité, dénommés "âmes". Les huit kikinu, par groupes de quatre dits de "corps" et de "sexe", sont les témoins de quatre êléments fondamentaux (air, eau, feu et terre), bases avec lesquelles le créateur réalisa I'univers. Sièges de la conscience et de la volonté de chaque individu,

(6) Marcel Griaule, Dieu d'eau, p. 70.

(7) Ibid., p. 164 .

Voir également Geneviève Calame Griaule, Ethnologie et Langage La parole chez les Dogon, Paris, Gallimard, 1965.

(8) Germaine Dieterlen, "Correspondances cosmo-biologiques chez les Soudanais", Joumal de psychologie normale et pathologique, $\mathrm{n}^{\circ} 3$, juil.-sept. 1950, p. 350-366. 
Nouvelles recherches sur le sacrifice chez les Dogon

ces principes ont autoritê sur la force vitale qu'ils contrôlent (9). Dans le cas de l'immolation d'une victime sacrificielle, lorsqu'ils sont libérés, ils retournent à leur créateur et, non plus que le nyoma de cette victime qu'ils dirigent et délèguent dans des buts précis, ils ne s'attaquent au sacrifiant ou aux agents' opérateurs.

Une étape importante fut franchie lorsque, abordant l'ensemble des concepts qui relèvent de la "connaissance profonde" et de 1'interprétation des rites par rapport à la cosmologie, les enquêtes eurent complètement dégagé les raisons, le schème, les modalités et les effets du sacrifice du Nommo, géniteur mythique des hommes, tels qu'ils ont été décrits dans Le Renard Pâte (10). Ce sacrifice est considéré comme essentiellement purificateur et réorganisateur : i1 intervient pour pallier les effets des désordres causés par le jumeau du Nommo, transformé en renard, et pour construire l'univers sur de nouvelles bases. Pour les Dogon, tout sacrifice actuel, si modeste soit-il, répète, reproduit celui du Nommo et, quel que soit son but, provoque des effets parallèles. L'étymologie dogon du terme qui désigne le sacrifice en général, bulu, est à elle seule révélatrice; bulu vient de bulo, "revivre, ressusciter". Le contenu claviculaire qui s'écoula au moment de 1 'opération, le sang du sexe, celui du cordon ombilical et celui du corps revivifièrent 1 'ensemble de 1 'univers en formation; le partage du corps en parcelles - que rappelle tout dépeçage actuel d'une victime sacrificielle - évoque "le travail d'Amma" réorganisant le monde sur de nouvelles bases par une distribution dans I'espace de ces parcelles; puis il les regroupa et ressuscita la victime.

Les recherches, menées selon la méthode que nous venons d'évoquer depuis la parution du premier volume du Renard Pâle et des publications que nous avons mentionnées, ont apporté des précisions sur les modalités des sacrifices et le sens que leur donnent les Dogon. Nous

(9) L'ensemble des composantes de la personne et de leurs interrelations ont êté exposêes dans : Germaine Dieterlen, L'image du corps et les composantes de la personne chez les Dogon, in La notion de personne en Afrique noire, Paris, C.N.R.S., 1973, p. 205-229.

(10) Germaine Dieterlen, Le Renard Pâle, Paris, Institut d'Ethnologie, 1965, chapitre III, p. 225-384. 
en donnerons ici quelques exemples qui, sur des plans différents, se rapportent au contenu des opérations sacrificielles et à leur interprétation.

Le premier exemple a trait aux effets purificateurs du sacrifice du Nommo. Un certain nombre de végêtaux naquirent du sang écoulé sur le placenta de la victime après qu'il fut séparé de son corps, lors de l'éviration, de l'égorgement, puis du dépeçage de ce même corps. Tous sont vivants, omo, car témoins de la purification effectuée par 1 'opération. Ces mêmes vêgétaux, particulièrement ceux qui sont associés aux organes internes de la victime et, parallèlement, aux "paroles" que véhicule le sang à travers ceux-ci, sont considérés comme des agents privilégiés et utilisés comme tels dans les rites de purification, qu'il s'agisse de purification d'individus, de groupes plus ou moins étendus, d'agglomérations, d'autels, de champs cultivés ou, dans la brousse, de lieux-dits associés symboliquement à des personnalités mythiques ou ancestrales et qui sont 1 'objet de rites réguliers. Il n'est pas possible d'aborder l'ensemble de ces opérations dans ce court préambule. Mais nous nous proposons, 1'an prochain, d'exposer en détail les noms, les buts, le contenu et les modalités des diverses et très nombreuses lustrations opérées par les Dogon qui, toutes, sont en relation étroite avec le schème évoqué ci-dessus et symboliquement en rapport avec les étapes du sacrifice primordial.

L'examen du contenu de certains rites agraires nous a permis de franchir une nouvelle étape. Les rites que 1 'on peut qualifier d"offrande des prémices" sont effectués, après les récoltes, sur divers autels collectifs ou familiaux et, notamment, sur celui qui symbolise les clavicules du sacrifié. L'opérateur verse successivement sur ce support :

-- une préparation crue, 'faite d'eau puisée dans la mare locale principale - où est censé vivre le Nommo ressuscitê - eau à laquelle on a mélangé de la farine de riz et de petit mil récoltés dans les champs de fonction des prêtres totêmiques;

-- une préparation cuite, faite d'eau et de graines de sorgho que 1 'on a préalablement fait germer pour en retirer les germes avant $1 \mathrm{a}$ cuisson. Le liquide refroidit, puis est cuit une seconde fois.

La première libation rappelle l'écoulement du contenu des clavicules du Nommo, ses buts et ses effets ; la seconde, 1'éviration, 
l'égorgement et la mort qui suivit.

Mais, dans le reste de la préparation cuite on mettra le support à ferment, image du placenta de la victime resté pur et vivant. La bière fermentée ainsi obtenue sera consommée par tous les participants après qu'une libation ait été effectuée sur la terre, près de l'aute1. La fermentation de la boisson est assimilée à la résurrection du grain tué par la cuisson et, parallèlement, à la résurrection du Nommo. Toutes les étapes de la fabrication de la bière évoquent symboliquement celles du sacrifice primordial (11).

Mais là ne se bornent pas les spéculations des Dogon et les explications qu'apportent les informateurs sur ces rites agraires. Les libations sont accompagnées d'une longue prière. Elles ont pour but non pas d'apporter au Nommo les prémices, mais en réintégrant les graines récoltées dans ses propres clavicules et en lui demandant de confier leurs "âmes" - les huit kỉkinu - à ses frères célestes, de les préserver de tout risque d'impureté. Il s'agit d'éviter toute perte de force vitale aux semences qui séjourneront à 1 'abri dans des greniers spéciaux jusqu'aux semailles qui suivront. Le déplacement des "âmes" des plantes cultivées aura lieu dans 1 'autre sens le jour où, en effectuant des opérations sacrificielles dans des conditions parallèles, les responsables des cultes demanderont au Nommo d'intercéder pour que leurs âmes soient rendues aux semences ; celles qui ont été mises à part seront distribuées à tous les cultivateurs qui les mélangeront avec les leurs avant de les mettre en terre. Dans le même temps, le Nommo sera sollicité de veiller sur l'intégrité des champs et de leur contenu jusqu'à la récolte suivante : ainsi le cycle sera clos.

Cette courte analyse que nous nous proposons d'exposer et de développer 1 'an prochain, permet cependant $d^{\prime}$ apporter des réserves à 1 'interprêtation gênéralement admise de 1 'expression "offrande des prêmices". On apporte certes les prémices au Nommo, mais il n'est pas le bénéficiaire du rite : il est invoqué au bénéfice des hommes, ses descendants. Il s'agit ici bien plutôt de mouvements déclenchés par les opérations décrites ci-dessus, qui concernent les principes

(11) Germaine Dieterlen, Amma boy, analyse d'une prière dogon, in l'Autre et l'Ailleurs - Hommage à Roger Bastide, Berger-Levrault, Paris, 1976, p. 248-272. 
spirituels des plantes cultivées ; I'action du Nommo est sollicitée par 1a "parole" de la prière énoncée à ce moment et par la manipulation du contenu de ces opérations qui rappelle symboliquement le schème de son propre sacrifice.

Il conviendra d'autre part de poursuivre l'analyse des sacrifices mythiques, tels qu'ils apparaissent dans la cosmogonie des Dogon. Les informations consignées dans Dieu d'ecu associent sous une forme très résumée, nous l'avons vu, l'immolation du Nommo et celle de 1 'ancêtre Lêbê. Il est certain que cette dernière, effectuée pour rêaliser une purification nécessaire, est présentée comme le parallèle terrestre de celle qui eut lieu au ciel-empyrée, avant la création des hommes. Mais les enquêtes récentes ont révélé un troisième sacrifice mythique, celui de 1 'ancêtre Dyongou Sérou, frère du Lébé. Comme le Lêbé, Dyongou Sérou ressuscita; mais il subit une seconde mort physique, celle-là définitive. Le Grand Masque, qui le représente et dans lequel se regroupent tous ses principes spirituels, est taillé lors des cérémonies soixantenaires du Sigui ; le culte dont il est 1 'objet rappelle ces événements et, conjointement, 1 'apparition de la mort qui devait frapper tous les êtres vivants (12). Le schème de ce troisième sacrifice mythique est très différent des deux autres.

Nous nous proposons, l'année prochaine, de procéder à une mise en parallèle de leurs diverses modalités, à des analyses comparatives et à l'étude des commentaires apportés par les Dogon sur ces épisodes fondamentaux de leur cosmologie.

(12) Germaine Dieterlen, "Les cêrémonies soixantenaires du Sigui", Africa, XLI, 1, janv. 1971, p. 1-11. 